\title{
Maintenance of the synthesis of large proteoglycans in anatomically intact murine articular cartilage by steroids and insulin-like growth factor I
}

Peter $M$ van der Kraan, Elly L Vitters, Nancy S Postma, Jeanine Verbunt, Wim B van den Berg

\begin{abstract}
Objectives-The exact regulation of the synthesis of cartilage specific molecules, such as collagen type II and aggrecan, by articular chondrocytes is unknown, but growth factors and hormones probably play an important part. The effects of glucocorticosteroids (prednisolone and triamcinolone), in combination with insulin-like growth factor I (IGF-I), on the synthesis and hydrodynamic volume of proteoglycans from murine patellar cartilage were investigated. Methods-The in vitro effect of IGF-I and steroids on proteoglycan synthesis in murine patellar cartilage was evaluated by $\left[{ }^{35} S\right]$ sulphate incorporation in combination with dissociative gel chromatography using a Sephacryl S-1000 column. The impact of in vivo prednisolone (0-5 mg/kg) on proteoglycan synthesis in murine patellar cartilage was analysed by $\left[{ }^{35} \mathrm{~S}\right] \mathrm{sul}$ phate incorporation immediately after dissection from the knee joint.

Results-Prednisolone stimulated proteoglycan synthesis in murine patellar cartilage from normal knees and in cartilage from knees injected with papain in vitro in the absence and presence of IGF-I. Moreover, oral administration of prednisolone for seven days to $\mathrm{C57 \textrm {B } 1 1 0}$ mice resulted in enhanced proteoglycan synthesis in patellar cartilage. The incubation of patellar cartilage for 48 hours without serum or growth factors led to the synthesis of proteoglycans with a smaller hydrodynamic volume than those synthesised immediately after dissection of the patellae. This could either be circumvented by the addition of IGF-I or by the addition of glucocorticosteroids (prednisolone or triamcinolone) to the culture medium.

Conclusions-These results show that in a dose range of $0.0003-0.3 \mathrm{mmol} / \mathrm{l}$, glucocorticosteroids, like IGF-I, stimulate proteoglycan synthesis and maintain the synthesis of hydrodynamically large proteoglycans by chondrocytes from murine articular cartilage. This indicates that glucocorticosteroids might play a part in the preservation of matrix integrity in articular cartilage.
\end{abstract}

(Ann Rheum Dis 1993; 52: 734-741)
Articular cartilage consists of chondrocytes embedded in an extracellular matrix rich in collagen type II and the hyaluronic acid binding, cartilage specific proteoglycan aggrecan. Aggrecan gives articular cartilage its unique ability to resist compression and to undergo reversible deformation, whereas collagen provides tensile strength. Control of chondrocyte biosynthetic function is essential in the maintenance and integrity of this tissue. Disturbances in the metabolism of the articular chondrocytes, as in osteoarthritis and arthritis, will lead to the deterioration of articular cartilage and eventually to joint failure. Insulin-like growth factor I (IGF-I) is considered to be a cardinal factor in the maintenance of chondrocyte biosynthetic function. This factor appears to be as effective as serum in stimulating proteoglycan synthesis in bovine, human, and murine chondrocytes. ${ }^{1-4}$ Although it has been reported that IGF-I has no effect on proteoglycan degradation in bovine explants, other workers have observed considerable inhibition of proteoglycan breakdown by IGF-I in bovine and murine cartilages. ${ }^{15-7}$

Glucocorticosteroid receptors have been shown to be present on articular cartilage chondrocytes, indicating that chondrocyte metabolism can be influenced directly by physiological and therapeutic glucocorticoids. $^{8}{ }^{9}$ The specific effects of glucocorticosteroids on chondrocyte proteoglycan synthesis are conflicting, however, and remain controversial. An inhibitory effect of glucocorticosteroids on proteoglycan synthesis has been reported for normal rat, bovine, and porcine chondrocytes $^{10-12}$ and human osteoarthritic cartilage. ${ }^{13}$ In contrast, the maintenance of differentiated chondrocyte phenotype and proteoglycan synthesis are stimulated in rabbit chondrocytes by glucocorticosteroids. ${ }^{14-16}$ Moreover, there appears to be a synergistic action of glucocorticosteroids and IGF-I on proteoglycan synthesis in rabbit chondrocytes. ${ }^{17}$

In this study we examined the effect of steroids, in the presence and absence of IGF-I, on proteoglycan synthesis in murine patellar cartilage. Patellar cartilage from normal knees and knees injected with papain was used. The injection of papain leads to a repair response in patellar cartilage mimicking 
the repair response observed in osteoarthritic cartilage. During in vitro culture of normal cartilage and of cartilage from knees injected with papain prednisolone stimulated the synthesis of proteoglycans at low and moderate concentrations $(0.0003-0.3 \mathrm{mmol} / \mathrm{l})$ in the presence and absence of IGF-I. Administration of prednisolone by mouth resulted in increased proteoglycan synthesis in patellar cartilage, as measured by $\left[{ }^{35} \mathrm{~S}\right]$ sulphate incorporation ex vivo. Furthermore, steroids and IGF-I were able to maintain the synthesis of normal proteoglycans during the in vitro culture of patellar cartilage in the absence of serum factors.

\section{Materials and methods}

INDUCTION OF A REPAIR RESPONSE IN ARTICULAR CARTILAGE

C57B110 mice (20-25 g, 10-16 weeks old) were used in all experiments. They were fed a commercial pellet diet (RHM, Hope Farms, Linschoten, The Netherlands) and given acidified tap water ad libitum. The animals were kept in boxes with sawdust bedding in an air conditioned room.

The induction of an articular cartilage repair response was performed as described previously. ${ }^{18}$ In brief, the right knee joints of mice were injected once, intra-articularly, with $6 \mu l 5$ $\mathrm{mg} / \mathrm{ml}$ papain (type IV, double crystallised, 15 $\mathrm{U} / \mathrm{mg}$; Sigma, St Louis, MO, USA) in a solution of $0.03 \mathrm{M}_{\mathrm{L}}$-cysteine hydrochloride (Sigma) to activate the papain. Three days after the injection of papain the patellae were dissected and used in the various experiments. This protocol results in a moderate depletion of patellar cartilage proteoglycans resulting in a stimulation of proteoglycan synthesis of approximately $50 \%$ above normal values on the third day after injection. ${ }^{18}$

EFFECT OF PREDNISOLONE ON PROTEOGLYCAN SYNTHESIS IN VITRO

Mice were killed by cervical dislocation and the whole patellae, with a standard amount of surrounding tissue, were dissected from normal knee joints and knee joints injected with papain according to the method of van den Berg et al. ${ }^{19}$ Patellae were incubated in the absence and presence of a for mice physiological concentration of IGF-I $(0.25 \mu \mathrm{g} / \mathrm{ml}$; Boehringer, Mannheim, Germany) in RPMI 1640 DM medium (Flow Laboratories, Irvine, UK) supplemented with various concentrations of prednisolone disodium phosphate $(0-3$ $\mathrm{mmol} / \mathrm{l}$; Sigma). All incubations were performed for 24 hours in 24 well cluster dishes at $37^{\circ} \mathrm{C}$ in a humidified atmosphere containing $5 \%$ carbon dioxide. The last two hours of the culture period were carried out in the presence of $1.48 \mathrm{MBq}\left[{ }^{35} \mathrm{~S}\right]$ sulphate $\left(\mathrm{Na}_{2} \mathrm{SO}_{4} ; \mathrm{Du}\right.$ Pont de Nemours, Den Bosch, The Netherlands).

After incubation the patellae were washed three times with physiological saline to remove non-incorporated radiolabel and were subsequently fixed in $96 \%$ ethanol. Decalcification of the patellae with $5 \%$ formic acid was followed by stripping of the articular cartilage layer from the remainder of the patella. Patellar cartilage was digested in Lumasolve (Perstorp Analytical, Oud-Beijerland, The Netherlands) at $60^{\circ} \mathrm{C}$. The amount of incorporated $\left[{ }^{35} \mathrm{~S}\right]$ sulphate was measured by liquid scintillation analysis.

EFFECT OF IGF-I AND STEROIDS ON HYDRODYNAMIC VOLUME OF NEWLY SYNTHESISED PROTEOGLYCANS

Murine patellae were dissected from normal knee joints and knee joints injected with papain. The patellae were incubated for 48 hours in the presence and absence of IGF-I $(0.25 \mu \mathrm{g} / \mathrm{ml})$ and various concentrations of prednisolone or triamcinolone acetonide (Sigma; 0, 0.03, and $0.3 \mathrm{mmol} / \mathrm{l}$ ). After incubation the patellae were cultured for four hours in RPMI 1640 DM medium containing $6 \mathrm{MBq}\left[{ }^{35} \mathrm{~S}\right]$ sulphate followed by a thorough rinsing in physiological saline. In additional experiments the labelling interval was followed by a 24 hour chase period in medium containing similar supplements to the original incubation medium.

Subsequently, the patellae were decalcified in $3.5 \% \mathrm{Na}_{2}$-EDTA (Merck, Darmstadt, Germany) for four hours and thereafter the cartilage layer was stripped from the bone. The stripped cartilage was ground with a glass mortar and pestle followed by proteoglycan extraction at $4^{\circ} \mathrm{C}$ for 24 hours with $4 \mathrm{M}$ guanidinium hydrochloride (Merck) in the presence of protease inhibitors $(0.01 \mathrm{M}$ EDTA, 0.1 M 6-aminocaproic acid (Sigma), $0.005 \mathrm{M}$ benzamidine hydrochloride (Sigma), $5 \mathrm{mg} / \mathrm{ml}$ trypsin inhibitor (Sigma), 0.005 M iodoacetate (Sigma)). This extraction procedure resulted in the extraction of over $90 \%$ of the incorporated radiolabel.

Extracted proteoglycans were supplemented with $1 \mathrm{mg}$ of an unlabelled bovine proteoglycan mixture as a carrier and applied to a Sephacryl S-1000 column $(1.6 \times 100 \mathrm{~cm}$, Pharmacia, Uppsala, Sweden). The column was equilibrated and eluted with $4 \mathrm{M}$ guanidinium chloride (dissociative). The flow rate was $6 \mathrm{ml} / \mathrm{h}$ and fractions of $3 \mathrm{ml}$ were collected. The fractions were assayed for radioactively labelled proteoglycans using a liquid scintillation counter. All runs were checked for reproducibility by comparison of the elution pattern of the unlabelled bovine carrier proteoglycans of different runs. All runs of one particular experiment were performed on the same column.

EFFECT OF IN VIVO PREDNISOLONE

ADMINISTRATION ON PROTEOGLYCAN

SYNTHESIS

Male C57Bl mice (12-16 weeks) were injected in the right knee joint with $6 \mu \mathrm{l}$ of $5 \mathrm{mg} / \mathrm{ml}$ papain as described earlier. The left knee was injected with a similar volume of physiological saline. Prednisolone (0-5 $\mathrm{mg} / \mathrm{kg}$ ) dissolved in tap water was administered by mouth daily to the mice for a period of seven days, starting one day after the injection of papain. Control animals received a comparable volume of tap 
water only. Groups of mice were matched for weight at the start of the experiment.

Mice were killed by cervical dislocation and the patellae were dissected. Patellae were cultured for two hours in medium containing $1.48 \mathrm{MBq}\left[{ }^{35} \mathrm{~S}\right]$ sulphate. After incubation the patellae were washed three times with physiological saline to remove non-incorporated $\left.{ }^{35} \mathrm{~S}\right]$ sulphate and were fixed in $96 \%$ ethanol. Decalcification of the patellae with $5 \%$ formic acid was followed by stripping of the articular cartilage layer. Patellar cartilage was digested in Lumasolve at $60^{\circ} \mathrm{C}$. The amount of incorporated $\left[{ }^{35} \mathrm{~S}\right]$ sulphate was measured by liquid scintillation analysis.

\section{STATISTICS}

Statistical evaluation of the in vitro experiments was tested by one-way analysis of variance in combination with Student's $t$ test. The in vivo experiments were evaluated by the Kruskal-Wallis test in combination with the Wilcoxon test. A p value less than $5 \%$ was considered significant.
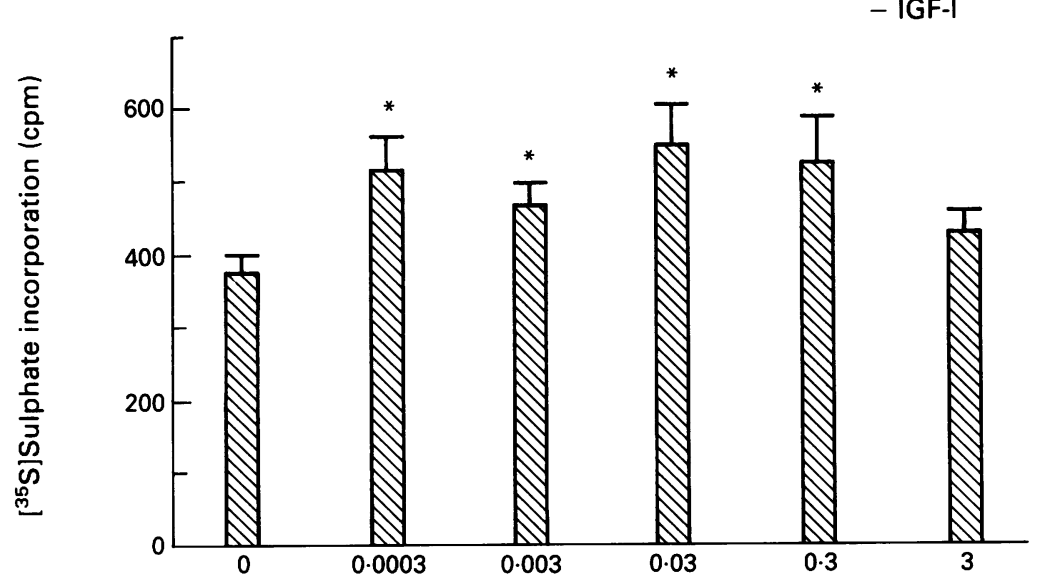

Prednisolone $(\mathrm{mmol} / \mathrm{l})$

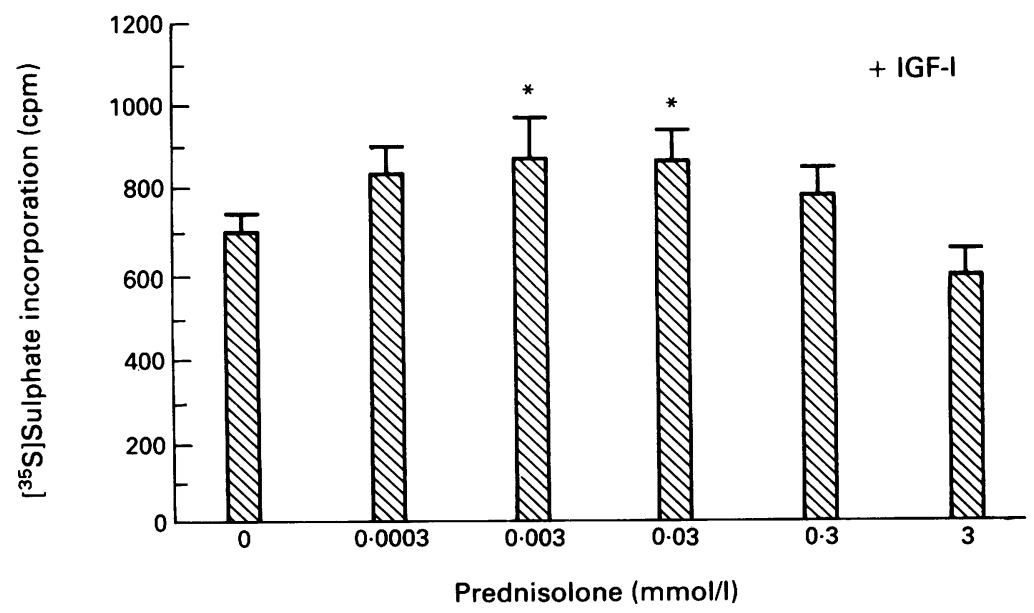

Figure 1 Effect of prednisolone on the incorporation of $\left.{ }^{35} S\right]$ sulphate in patellar cartilage from normal joints. Patellae were incubated in the absence and presence of insulin-like from normal joints. Patellae were incubated in the absence and presence of insulin-like
growth factor I (IGF-I) $(0.25 \mu \mathrm{g} / \mathrm{ml})$ with various concentrations of prednisolone for 24 hours. The last two hours of the culture period were carried out in the presence of $\left.{ }^{35} S\right]$ sulphate. Results are the mean $(S E M)$ of 21 samples $\left({ }^{\star} p<0 \cdot 05\right)$. Statistical significance was analysed with respect to the control group after one way analysis of variance.

\section{Results}

EFFECT OF PREDNISOLONE ON PROTEOGLYCAN SYNTHESIS IN VITRO

Immediately after dissection the proteoglycan synthesis in patellar cartilage of knee joints injected with papain, measured by $\left[{ }^{35} \mathrm{~S}\right] \mathrm{sul}-$ phate incorporation, was $55 \%$ higher than in the patellar cartilage of the control joints injected with saline. In vitro this enhanced proteoglycan synthesis was lost within 24 hours. The addition of IGF-I to the culture medium resulted in a significantly increased proteoglycan synthesis in normal cartilage and cartilage from knee joints injected with papain compared with proteoglycan synthesis without IGF-I (approximately 90\%). The proteoglycan synthesis in the presence of IGF-I was comparable with the in vivo level of proteoglycan synthesis (data not shown).

Prednisolone had a significant stimulating effect on patellar proteoglycan synthesis in the absence and presence of IGF-I during a 24 hour incubation period (figs 1 and 2). A linear dose-response relation was not observed, but prednisolone had a Gaussianlike effect on the synthesis of proteoglycans. In the absence of IGF-I, concentrations ranging from 0.0003 to $0.3 \mathrm{mmol} / 1 \mathrm{had}$ a significant stimulating effect on proteoglycan synthesis in patellar cartilage from normal knees and knees injected with papain (maximum $47 \%$ ). In the presence of IGF-I, a stimulating effect of prednisolone was observed at 0.003 and 0.03 $\mathrm{mmol} / \mathrm{l}$ with normal patellae and at 0.0003 , 0.003 , and $0.3 \mathrm{mmol} / 1$ with patellae from murine knee joints injected with papain. A concentration of $3 \mathrm{mmol} / \mathrm{l}$ led to neither a significant stimulation nor inhibition of proteoglycan synthesis.

EFFECT OF IGF-I AND STEROIDS ON HYDRODYNAMIC VOLUME OF NEWLY SYNTHESISED PROTEOGLYCANS

The culture of patellae from normal knee joints for 48 hours in medium free of serum and growth factor resulted in the synthesis of proteoglycan monomers with a smaller hydrodynamic volume than the proteoglycans synthesised immediately after dissection (ex vivo labelling; fig 3 ). The addition of IGF-I to the medium maintained the synthesis of proteoglycans with a hydrodynamic volume similar to those synthesised directly after dissection. These results indicate that the addition of IGF-I to the culture medium results in the synthesis of 'normal' patellar cartilage proteoglycans during in vitro incubation. In this context normal means similar to the proteoglycans synthesised ex vivo. The murine proteoglycan monomer (Kav 0.43 ) synthesised immediately after dissection appeared to be smaller than monomers from bovine articular cartilage ( $\operatorname{Kav} 0 \cdot 25$, data not shown).

The effect of prednisolone mimicked the effect of IGF-I on the hydrodynamic volume of newly synthesised proteoglycans (fig 4). In cultures with no IGF-I the addition of 0.03 or $0.3 \mathrm{mM}$ prednisolone led to the production of 

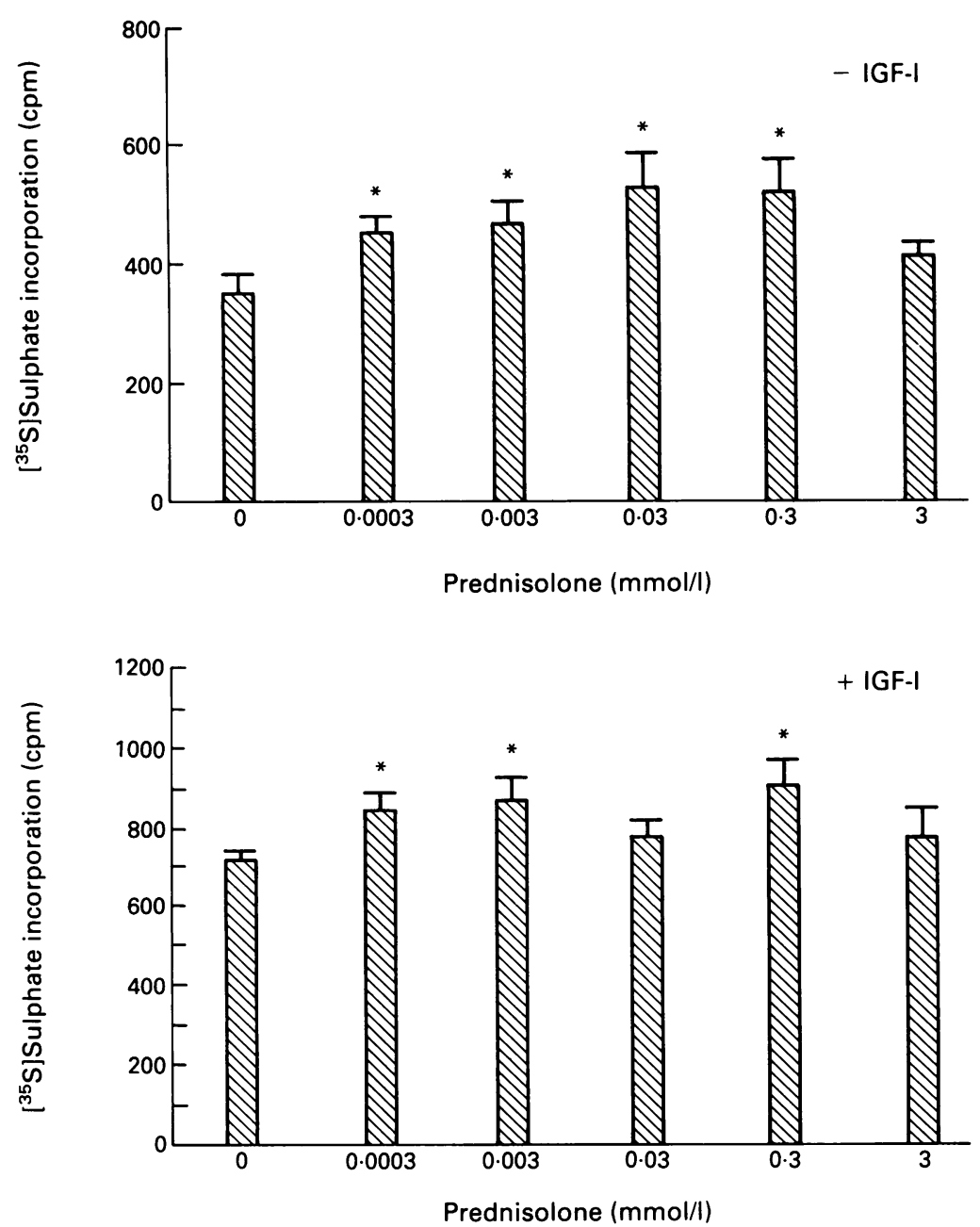

Figure 2 Effect of prednisolone on the incorporation of ${ }^{35} S J$ sulphate in patellar cartilage from knee joints injected with papain. Patellae were incubated in the absence and presence of insulin-like growth factor I (IGF-I) $(0.25 \mu \mathrm{g} / \mathrm{ml})$ with various concentrations of

prednisolone for 24 hours. The last two hours of the culture period were carried out in the presence of $\left.{ }^{\beta 5} S\right]$ sulphate. Results are the mean (SEM) of 21 samples $\left({ }^{*} p<0.05\right)$.

Statistical significance was analysed with respect to the control group after one way analysis of variance.

proteoglycans with a hydrodynamic volume comparable with those synthesised ex vivo. In the presence of $0.03 \mathrm{mmol} / \mathrm{l}$, however, some of the synthesised proteoglycans were still smaller than the proteoglycans produced normally. Prednisolone had no effect on the hydrodynamic volume of the proteoglycans in the presence of IGF-I (fig 4).

As expected, the effect of prednisolone on the dimension of newly synthesised proteoglycans did not appear to be specific to prednisolone. Triamcinolone acetonide had effects analogous to prednisolone on the hydrodynamic volume of newly synthesised proteoglycans (fig 5). Concentrations of 0.03 and 0.3 $\mathrm{mM}$ triamcinolone enhanced the synthesis of hydrodynamically large proteoglycans by patellar cartilage cultured in the absence of IGF-I (fig 5A). In the presence of IGF-I, no effects of triamcinolone were detectable on the proteoglycan hydrodynamic volume (fig 5C).

During a 24 hour chase period approximately $50 \%$ of the proteoglycans was lost in the absence of IGF-I, whereas this was only $20 \%$ in the presence of IGF-I. Triamcinolone had no effect on proteoglycan loss during the 24 hour chase period. The

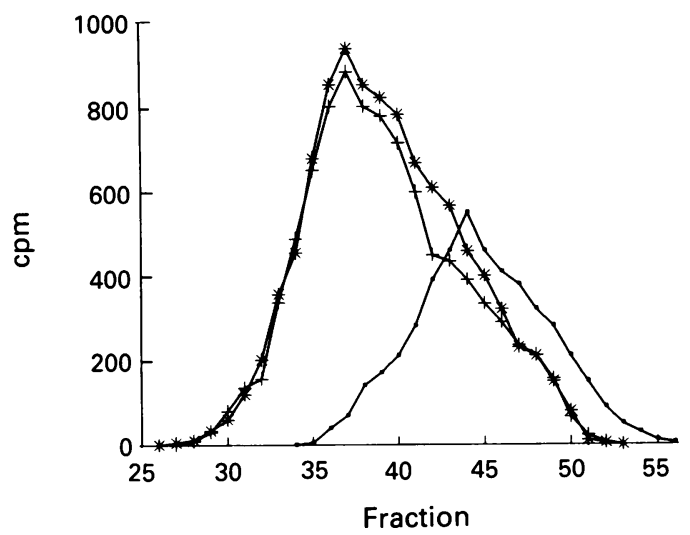

Figure 3 Effect of in vitro culture on hydrodynamic volume of newly synthesised proteoglycans of murine patellar cartilage in the presence and absence of insulin-like growth factor I (IGF-I). Comparison with proteoglycans synthesised ex vivo. Proteoglycans were radiolabelled with $\left.{ }^{35} S\right]$ sulphate for four hours immediately after dissection or after 48 hours of incubation with or without IGF-I. Proteoglycans were applied to a Sephacryl S-1000 column and eluted under dissociative conditions. ${ }^{\star}=E x$ vivo; $+=$ with IGF-I; $\bullet=$ without IGF-I. One representative experiment of four is shown.
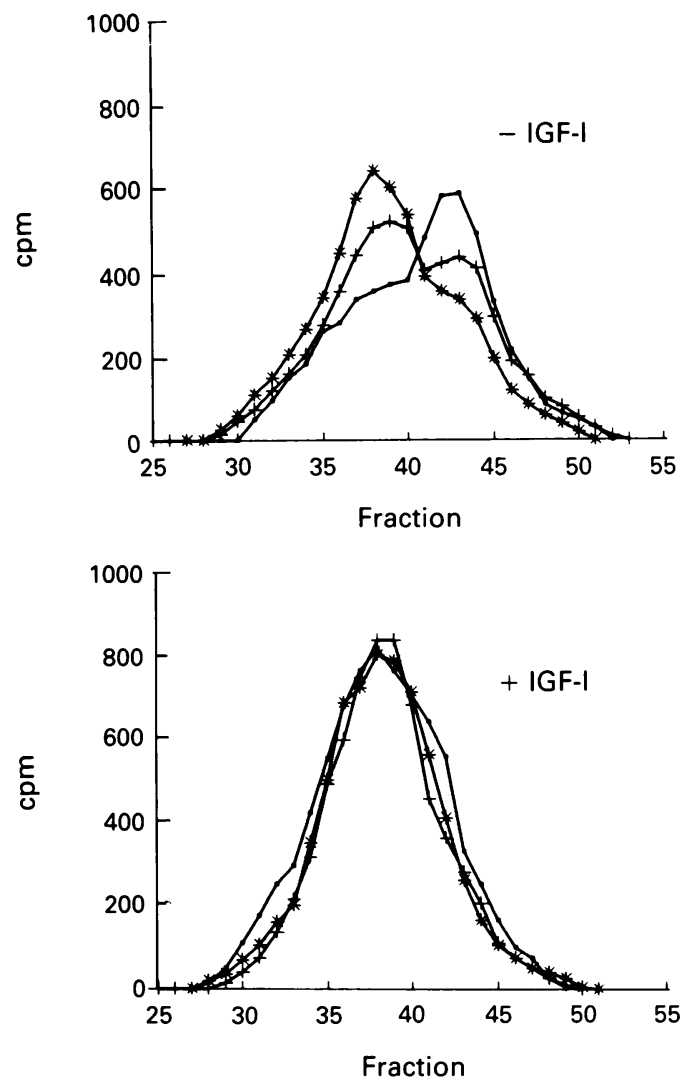

Figure 4 Effect of prednisolone on hydrodynamic volume of newly synthesiscd proteoglycans of normal patellar cartilage, in the presence and absence of insulin-like growth factor I (IGF-I). Proteoglycans were radiolabelled with ${ }^{35} S 7$ sulphate for four hours after 48 hours of incubation with prednisolone. Proteoglycans were applied to a

Sephacryl S-1000 column and eluted under dissociative conditions. $\bullet=0 \mathrm{~mol} / ;+=0.03 \mathrm{mmol} /$; and ${ }^{\star}=0.3$ mmoll prednisolone. One representative experiment of four is shown.

chromatographic profiles obtained immediately after labelling and after a 24 hour chase period were essentially similar (fig $5 B$ and $D$ ). Only a slight shift to a larger hydrodynamic volume of the proteoglycans was observed. 

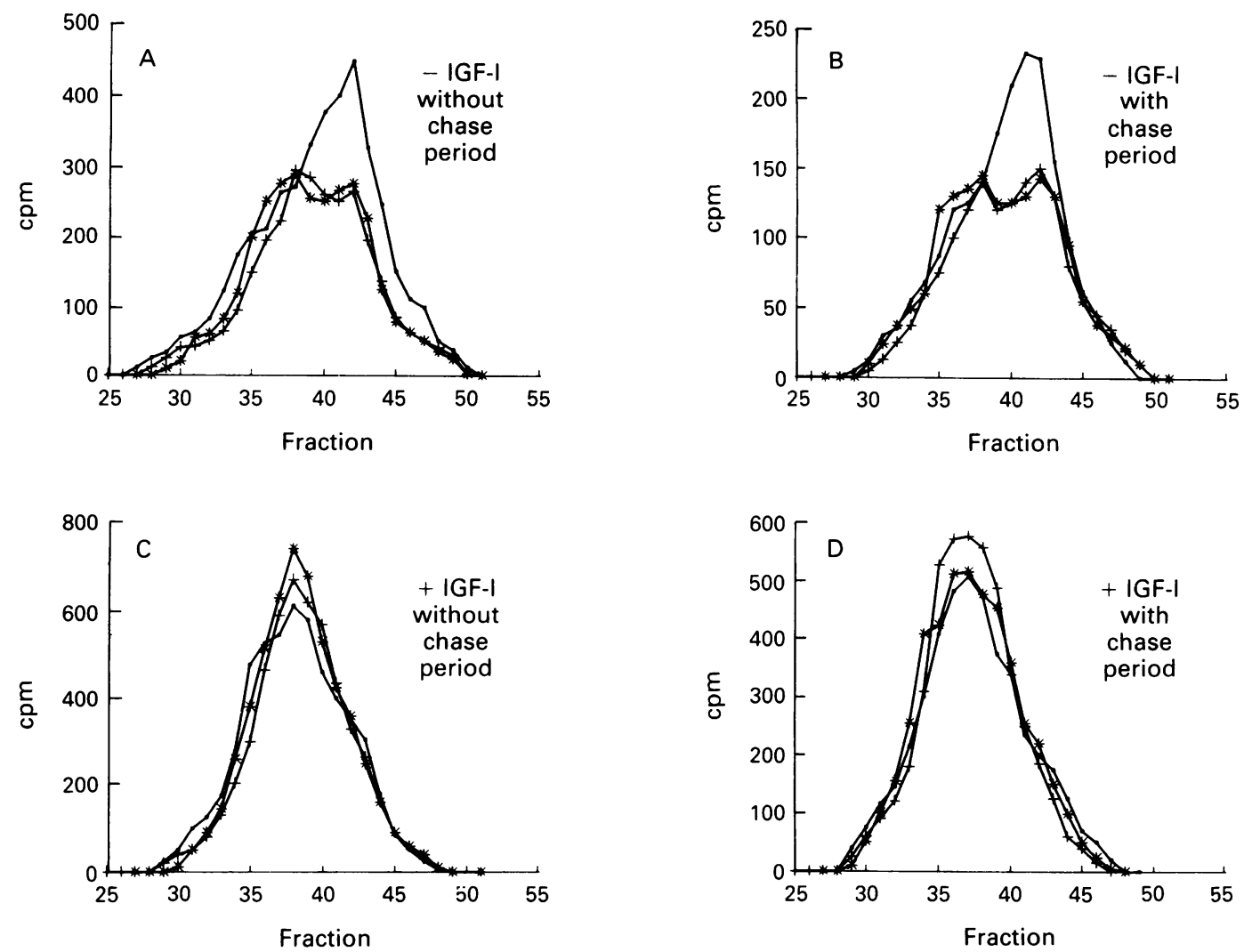

Figure 5 Effect of triamcinolone acetonide on hydrodynamic volume of newly synthesised proteoglycans of normal patellar cartilage in the presence and absence of insulin-like growth factor I (IGF-I). Proteoglycans were radiolabelled with $\left.{ }^{35} S\right]$ sulphate for four hours after 48 hours of incubation with triamcinolone. Part of the patellae was subsequently incubated for another 24 hour (chase). Proteoglycans were applied to a Sephacryl S-1000 column and eluted under dissociative conditions. $\bullet=0 \mathrm{mmol} /+=0.03 \mathrm{mmol} ;$; and ${ }^{\star}=0.3 \mathrm{mmol}$ triamcinolone acetonide.

This is probably caused by a preferential loss of relatively small proteoglycans to the culture medium. During the chase period the chromatographic pattern was not changed from an 'IGF-like' to a 'non-IGF-like' pattern, indicating that the effects of steroids and IGF-I on the production of the two different proteoglycan populations was an effect on proteoglycan synthesis and not to be mediated by a decreased proteoglycan breakdown. In a separate set of experiments of patellae incubated without IGF-I and steroids and without a chase period, variation of the proteoglycan labelling interval from one to four hours resulted in similar chromatographic patterns, illustrating that the effect of steroids and IGF-I was on newly synthesised proteoglycans instead of being caused by steroid and IGF-I induced alterations in proteoglycan degradation (data not shown). If proteoglycan breakdown affected the differences observed between IGFI/steroid and non-IGF/steroid chromatographic patterns a change in the elution profile dependent on the labelling period would be expected in the absence of IGF/steroids.

Patellar cartilage from knee joints injected with papain responded in a similar mannei to IGF-I and steroids as cartilage from normal knee joints (fig 6). Insulin growth factor I and triamcinolone maintained the synthesis of hydrodynamically large proteoglycans during a 48 hour culture period. In the presence of IGF-I triamcinolone had no additional effects on the dimensions of the newly synthesised proteoglycans.
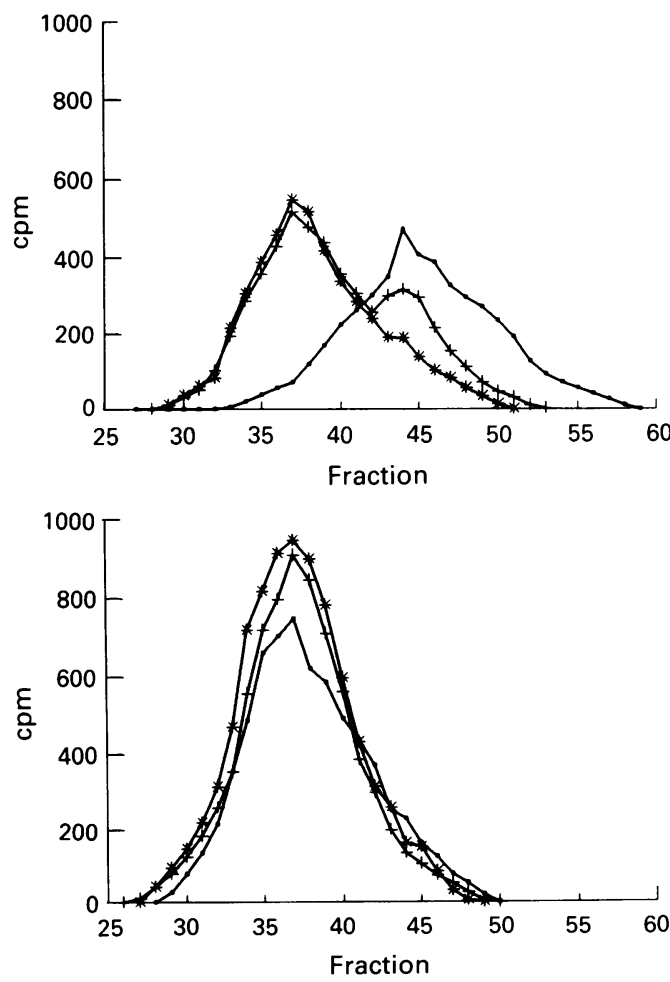

Figure 6 Effect of triamcinolone acetonide on hydrodynamic volume of newly synthesised proteoglycans of patellar cartilage from knee joints injected with papain in the presence and absence of insulin-like growth factor I (IGF-I). Proteoglycans were radiolabelled with

(IGF-I). Proteoglycans were radiolabelled with
$\left.{ }^{35} S\right]$ sulphate for four hours after 48 hours of incubation with triamcinolone. Proteoglycans were applied to a Sephacryl S-1000 column and eluted under dissociative conditions. $\bullet=0 \mathrm{mmol} /+=0.03 \mathrm{mmol} /$; and ${ }^{\star}=0.3$ mmoll triamcinolone acetonide. 
EFFECT OF IN VIVO PREDNISOLONE ADMINISTRATION ON PROTEOGLYCAN SYNTHESIS

Knee joints injected with papain showed a significantly enhanced (36\%) incorporation of radiolabelled sulphate in the patellar cartilage eight days after injection. Administration of prednisolone by mouth resulted in a significantly increased synthesis of proteoglycans at all doses $(0.5-5.0 \mathrm{mg} / \mathrm{kg})$ in normal cartilage and in cartilage with a repair response (fig 7) A dose-response effect of prednisolone on proteoglycan synthesis could not be shown in the dose range studied.

\section{Discussion}

Insulin-like growth factor I stimulated the synthesis of proteoglycans in murine patellar cartilage, as measured by $\left[{ }^{35} S\right]$ sulphate incorporation, and maintained the synthesis of normal (ex vivo-like) proteoglycans during in vitro culture. No differences were detected between normal cartilage and cartilage from knee joints injected with papain, indicating that cartilage with a subnormal proteoglycan

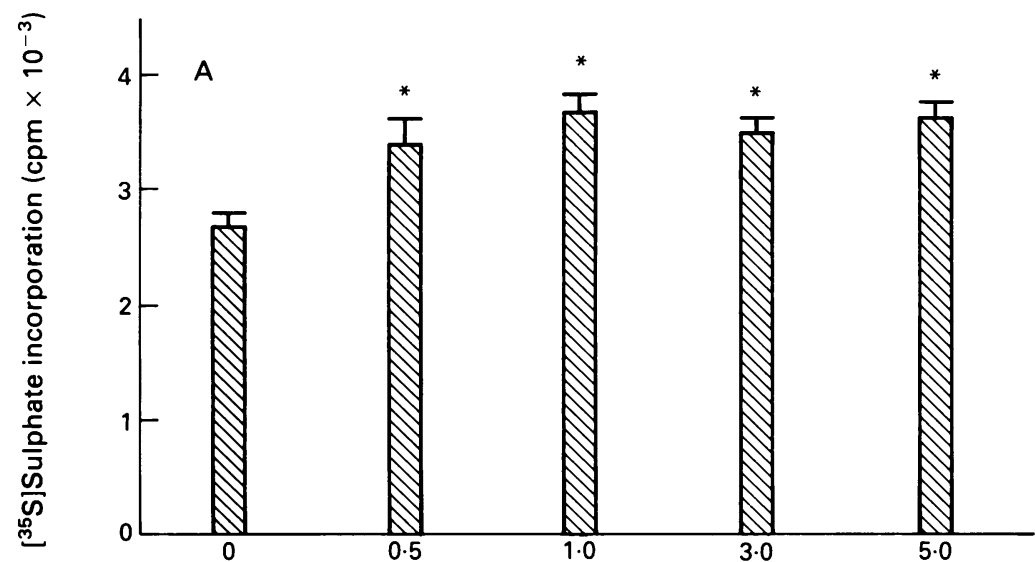

Prednisolone $(\mathrm{mg} / \mathrm{kg})$
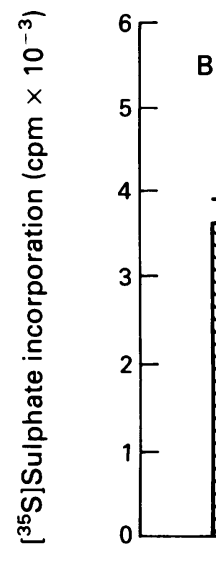

B
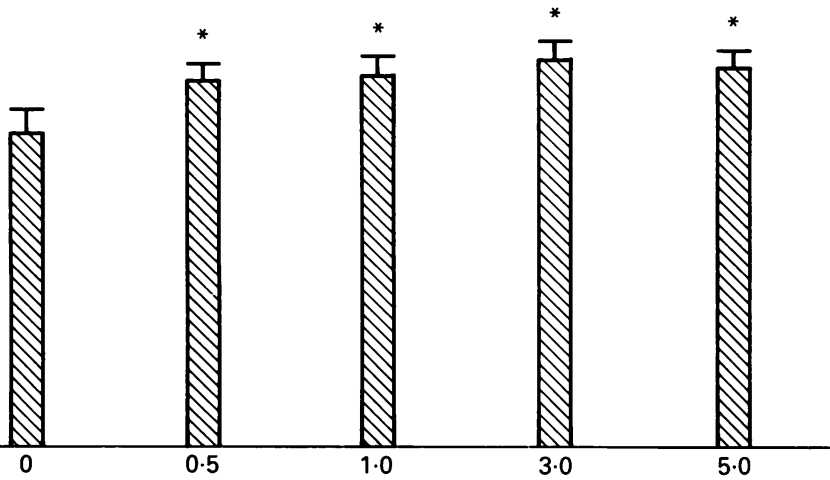

Prednisolone $(\mathrm{mg} / \mathrm{kg})$

Figure 7 Effect of oral administration of prednisolone for seven days on the ex vivo proteoglycan synthesis of patellar cartilage from normal knee joints and knee joints injected with papain. The right knee joint was injected with $6 \mu \mathrm{l} 5 \mathrm{mg} / \mathrm{ml}$ papain whereas the lef knee joint was injected with a similar volume of physiological saline. Prednisolone $(0-5 \mathrm{mg} /$ $\mathrm{kg}$ ) dissolved in tap water was given by mouth to the mice daily for a period of seven days, starting one day after the injection of papain. Control animals received a comparable volume of tap water only. After dissection patellae were incubated for two hours in ${ }^{35} S$ Jsulphate. Results are means (SEM) of 27-35 samples. (A) Normal joints; (B) joints injected with papain. ${ }^{\star} p<0.05$. Statistical significance was analysed with respect to the control group after one way analysis of variance. concentration and an enhanced proteoglycan synthesis rate was as responsive to IGF-I as normal cartilage. The hydrodynamic volume of the subpopulation of large proteoglycans of murine patellar cartilage appeared to be smaller than that of proteoglycans from bovine articular cartilage (our results, data not shown). This appears to be a general phenomenon. A smaller size of murine monomers compared with monomers from comparable tissues of larger species has been reported for proteoglycans from murine articular cartilage and from murine intervertebral disc. ${ }^{2021}$ The exact nature of the small molecular weight proteoglycans synthesised by patellar cartilage cultured without IFG-I, probably biglycan or decorin, is under study.

Other workers have reported that IGF-I preferentially stimulates the synthesis of large proteoglycans by chondrocytes. In experiments with rat rib growth plate chondrocytes, incubation with IGF-I resulted in an increased size of the newly synthesised proteoglycan monomers. ${ }^{22}$ In contrast, other growth factors such as epidermal growth factor and fibroblast growth factor favoured the synthesis of small proteoglycans. ${ }^{22}$ In bovine articular cartilage explants, IGF-I only stimulated the synthesis of the large proteoglycan subpopulation, but had no effect on the synthesis of small proteoglycans. ${ }^{23}$ These observations suggest that IGF-I is able to prevent the dedifferentiating effect of prolonged in vitro incubation when judged by the synthesis of proteoglycans.

Incubation of patellar cartilage for 24 hours with rather high concentrations of prednisolone $(0.0003-0.3 \mathrm{mmol} / \mathrm{l})$ resulted in a stimulation of $\left[{ }^{35} \mathrm{~S}\right]$ sulphate incorporation and only at a concentration of $3 \mathrm{mmol} / \mathrm{l}$ was no stimulation or only a slight, insignificant inhibition seen. With respect to the rate of proteoglycan synthesis, no significant difference in prednisolone effects was observed between cultures with or without IGF-I, neither with normal cartilage nor with cartilage from knees injected with papain. Moreover, in vivo administration of prednisolone up to the highest dose tested ( $5 \mathrm{mg} / \mathrm{kg}$ ) led to a significant stimulation of ex vivo proteoglycan synthesis in cartilage from normal knees and knees injected with papain. This is in agreement with the observation that proteoglycan synthesis in isolated rabbit chondrocytes was stimulated by glucocorticosteroids. ${ }^{14-16}$ In earlier studies with anatomically intact murine cartilage it was found that $0.3 \mathrm{mM}$ prednisolone had no significant effect on proteoglycan synthesis in normal cartilage, but stimulated proteoglycan synthesis in trypsin depleted cartilage and in cartilage from knee joints injected with zymozan. ${ }^{24}$

In contrast with these observations, an inhibiting effect of glucocorticosteroids on proteoglycan synthesis in articular cartilage has been shown by others. Dekel et al observed an inhibitory effect of hydrocortisone, at a concentration of $3 \mathrm{mmol} / \mathrm{l}$ and higher, on proteoglycan synthesis in cultured pig costal cartilage discs. ${ }^{10}$ In a minority of experiments, 
however, a stimulating effect of hydrocortisone was seen. ${ }^{10}$ In vitro culture with hydrocortisone decreased $\left[{ }^{35} \mathrm{~S}\right]$ sulphate incorporation in rat rib cartilage and human osteoarthritic cartilage, whereas dexamethasone had similar effects on pig cartilage. ${ }^{121325}$ Administration of prednisolone in rabbits and intra-articular injection of methylprednisolone in horses led to a decreased proteoglycan content and synthesis in articular cartilage. ${ }^{26}{ }^{27}$ In mice, intra-articular injection of rimexolone or triamcinolone hexacetonide suppressed proteoglycan synthesis in normal cartilage but, remarkably, increased proteoglycan synthesis in cartilage from arthritic knee joints. ${ }^{28-30}$

The background to these controversial effects remains obscure, but differences in steroid concentrations, low concentrations stimulating proteoglycan synthesis and high concentrations inhibiting proteoglycan synthesis, could be of major importance. In our hands moderate concentrations $(3 \mathrm{mM}$ prednisolone in vitro, $5 \mathrm{mg} / \mathrm{kg}$ by mouth) had no deleterious effects on proteoglycan synthesis. Species differences appear not be the determining factor as stimulating effects of steroids have been observed in murine, porcine, and rabbit cartilage. Steroid concentration in combination with the species studied could be of major importance, however. Also, the environment of the cartilage, normal or arthritic knee joint, seems to influence the effect of steroids on chondrocyte metabolism. ${ }^{28-30}$

Surprisingly, in anatomically intact patellar cartilage glucocorticosteroids not only had an increasing effect on proteoglycan synthesis but also preferentially stimulated the synthesis of proteoglycans with a large hydrodynamic volume. Corresponding effects have been observed by Kato and Gospodarowicz with isolated rabbit costal chondrocytes. ${ }^{15}$ Rabbit chondrocytes maintained in glucocorticoidfree medium produced high and low molecular weight proteoglycans, whereas chondrocytes in hydrocortisone supplemented medium synthesised almost only proteoglycans with a high molecular weight. ${ }^{15}$ Our results show that glucocorticosteroids, like IGF-I, preserve the differentiated chondrocyte phenotype, not only in cultures of isolated chondrocytes but also in anatomically intact cartilage.

Steroids appear, like IGF-I, to be able to maintain the synthesis of normal proteoglycans in cartilage from normal knees and in cartilage in a repair phase. No deleterious effects on proteoglycan synthesis in murine cartilage could be shown in this study, neither in vitro nor in vivo. These results indicate that glucocorticosteroids could play a part in the maintenance of the homeostasis and integrity of normal and disease affected articular cartilage. Full understanding of glucocorticosteroid effects on articular cartilage demands further research, however.

This study was supported by the Dutch League Against Rheumatism 'Nationaal Reumafonds'. The authors thank the öof the Central Animal Laboratory for their assistance in the experiments.
1 McQuillan D J, Handley C J, Campbell M A, Bolis S, Milway V E, Herington A C. Stimulation of proteoglycan synthesis by serum and insulin-like growth factor-1 in cultured bovine articular cartilage. Biochem $\mathcal{f} 1986 ; 240$ 424-30.

2 Recklies A D, Rougley P J, Bilmoria K M. Differentia response of young and old human articular chondrocytes to IGF-I and TGF $\beta$ [abstract]. Orthop Res Soc Tran 1989; 14: 281 .

3 Schalkwijk J, Joosten L A B, van den Berg W B, van Wijk J J, van de Putte L B A. Insulin-like growth facto stimulation of chondrocyte proteoglycan synthesis by human synovial fluid. Arthritis Rheum 1989; 32: 66-71.

4 Schalkwijk J, Joosten L A B, van den Berg W B, van de Putte L B A. Chondrocyte nonresponsiveness to insulin-like growth factor 1 in experimental arthritis. Arthritis Rheum 1989; 32: 894-900.

5 Luyten F P, Hascall V C, Nissley S P, Morales T I, Redd A H. Insulin-like growth factors maintain steady-stat metabolism of proteoglycans in bovine articular cartilage explants. Arch Biochem Biophys 1988; 267: 1-10

6 Barone-Varelas J, Schnitzer T J, Meng Q, Otten L, Thona E J M. Age-related differences in the metabolism of proteoglycans in bovine articular cartilage explants maintained in the presence of insulin-like growth factor I. Connect Tissue Res 1991; 26: 101-20.

7 van der Kraan P M, Vitters E L, van den Berg W B. Inhibition of proteoglycan synthesis by transforming growth factor $\beta$ in anatomically intact articular cartilage of murine patellae. Ann Rheum Dis 1992; 51: 643-7.

8 Blondelon D, Adolphe M, Zizine L, Lechat P. Evidence fo glucocorticoid receptors in cultured rabbit articular glucocorticoid receptors in cultured rab
chondrocytes. FEBS Lett 1980; 117: 195-8.

9 DiBattista J A, Martel-Pelletier J, Wosu L O, Sandor T, Antakly T, Pelletier J P. Glucocorticosteroid recepto mediated inhibition of interleukin-1 stimulated neutra metalloprotease synthesis in normal human chondrocytes. $\mathcal{f}$ Clin Endocrinol Metab 1991; 72: 316-26.

10 Dekel S, Felcorner J, Francis M J O. The effect of antiinflammatory drugs on glycosaminoglycan sulfation in pig cartilage. Prostaglandins Med 1980; 4: 133-40.

11 Kolibas L M, Goldberg R L. Effects of cytokines and antiinflammatory drugs on glycosaminoglycan synthesis by bovine articular chondrocytes. Agents Actions 1989; 27: 245-9.

12 Rayan V, Hardingham T E. Proteoglycan metabolism in chondrocytes of young pig articular cartilage and its chondrocytes of young pig articular cartilage and its
modulation by therapeutic agents. Biochem Soc Tran 1990; 643: 972

13 Pelletier J P, Cloutier J M, Martel-Pelletier J. In vitro effects of tiaprofenic acid, sodium salicylate and hydrocortison on the proteoglycan metabolism of human osteoarthriti cartilage. F Rheumatol 1989; 16: 646-55.

14 Takano T, Takigawa M, Suzuki F. Stimulation of glucocorticoids of the differentiated phenotype of chondrocytes and the proliferation of rabbit costal chondrocytes in culture. F Biochem 1985; 97: 1093-100.

15 Kato Y, Gospodarowicz D. Stimulation by glucocorticoid of the synthesis of cartilage-matrix proteoglycans produced by rabbit costal chondrocytes in vitro. $\mathcal{F}$ Biol Chem 1985 260: 2364-73.

16 Takigawa M, Takano T, Nakagawa K, Sakuda M, Suzuk F. Hydrocortisone stimulation of proliferation and F. Hydrocortisone stimulation of proliferation and chondrocytes in vitro. Arch Oral Biol 1988; 33: 893-9.

17 Itagane $Y$, Inada $\mathrm{H}$, Fujita $\mathrm{K}$, Isshiki $\mathrm{G}$. Interaction between steroid hormones and insulin-like growth factor-I in rabbit chondrocytes. Endocrinology 1991; 128: 1419-24.

8 van der Kraan P M, Vitters E L, van de Putte L B A, va den Berg W B. Development of osteoarthritic lesions in mice by "metabolical" and "mechanical" alterations in the knee joints. Am f Pathol 1989; 135: 1001-14.

19 van den Berg W B, Kruijsen M W M, van de Putte L B A. The mouse patella assay. An easy method of quantitating articular cartilage chondrocyte function in vivo and in vitro. Rheumatol Int 1982; 1: 165-9.

20 Venn G, Mason R M. Biosynthesis and metabolism in vivo of intervertebral-disc proteoglycans in the mouse. Biocher o 1983; 215: 217-25.

21 Rostand K S, Baker J R, Caterson B, Christner J E. Isolation and characterization of mouse articular cartilage proteoglycans using preformed $\mathrm{CsCl}$ density gradients in proteoglycans using preformed CsCl density gradients

22 Makower A M, Wroblewski J, Pawlowski A. Effects of IGF I, EGF, and FGF on proteoglycan synthesized by fractionated chondrocytes of rat rib growth plate. $\operatorname{Exp} C e$ Res 1988; 179: 498-506.

23 Tesch G H, Handley C J, Cornell $\mathrm{H} \mathrm{J}$, Herington A C. Effects of free and bound insulin-like growth factors on proteoglycan metabolism in articular cartilage explants. Orthop Res 1992; 10: 14-22.

4 De Vries B J, van den Berg W B, Vitters E, van de Putte L B A. Effects of NSAIDs on the metabolism of sulphated glycosaminoglycans in healthy and (post) arthritic murine articular cartilage. Drugs 1988; 35: (suppl 1): 24-32.

25 Boström H, Berntsen K, Whitehouse M W. Biochemica effects of anti-inflammatory drugs. II. Some effects on sulphate- ${ }^{35} \mathrm{~S}$ metabolism in vivo. Biochem Pharmacol 1964; 13: 413-20.

26 Hunneyball I M. Some further effects of prednisolone and triamcinolone hexacetonide on experimental arthritis in rabbits. Agents Actions 1981; 11: 490-8.

27 Chunekamrai S, Krook L P, Lust G. Maylin G A. Changes 
in articular cartilage after intra-articular injections of methylprednisolone acetate in horses. Am F Vet Res 1989 50: $1733-41$

28 Joosten L A B, Helsen M M A, van den Berg W B.

Protective effect of rimexolone on cartilage damage in arthritic mice: a comparative study with traimcinolon hexacetonide. Agents Actions 1990; 31: 135-42.

29 van den Berg W B. Impact of NSAID and steroids on cartilage destruction in murine antigen induced arthritis. f Rheumatol 1991; 18 (suppl 27): 122-3.

30 van den Berg W B, Joosten L A B, van de Loo A J, de Vries $B$ J, van der Kraan P M, Vitters E L. Drug evaluation on normal and arthritic mouse patellas. In: Kuettner $\mathrm{K} \mathrm{E}$ Schleyerbach R, Peyron J G, Hascall V C, eds. Articular cartilage and osteoarthritis. New York: Raven Press, 1991:583-96. 